\title{
Application of Static Adaptive Grid Techniques for Regional-Urban Multiscale Air Quality Modeling
}

\author{
Daewon Byun ${ }^{1}$, Peter Percell ${ }^{1}$, and Tanmay Basak ${ }^{2}$ \\ ${ }^{1}$ Institute for Multidimensional Air Quality Studies, \\ 312 Science Research Bldg. University of Houston, Houston, Tx 77204-5007 \\ \{dwbyun, ppercell\} @math.uh.edu \\ http: / / www. imaqs. uh. edu \\ ${ }^{2}$ Department of Chemical Engineering, I.I.T. Madras, Chennai - 600 036, Inida \\ tanmay@itm.ac.in
}

\begin{abstract}
Texas Air Quality Study 2000 revealed that ozone productivity in the Houston Ship Channel area was abnormally higher than other comparable cities in USA due to the large emissions of highly reactive unsaturated hydrocarbons from petrochemical industries. Simulations with popular Eulerian air quality models were shown to be inadequate to represent the transient high ozone events in the Houston Ship Channel area. In this study, we apply a multiscale Eulerian modeling approach, called CMAQ/SAFE, to reproduce the measured ozone productivity in the Houston Ship Channel and surrounding urban and rural areas. The modeling tool provides a paradigm for the multiple-level regional and local air quality forecasting operations that can utilize modern computational infrastructure such as grid computing technologies allowing to harness computing resources across sites by providing programmatic and highbandwidth data linkage and establishing operational redundancy in the case of hardware or software failures at one operational site.
\end{abstract}

\section{Introduction}

Air quality in the Houston area suffers from high ozone levels. It is aggravated, by the considerable amounts of emissions of Volatile Organic Compounds (VOCs) from chemical processing plants distributed along the Houston Ship Channel and mobile $\mathrm{NO}_{\mathrm{x}}\left(\mathrm{NO}\right.$ and $\mathrm{NO}_{2}$ ) emissions from the traffics in the metropolitan area. In the presence of sunlight, VOCs and $\mathrm{NO}_{\mathrm{x}}$ react in complex ways to form ozone. These concentrated local emissions (typically within sub-domains of size less than $4 \mathrm{~km}$ ) pose a challenge for existing computational models such as the EPA Models-3 Community Multiscale Air Quality Modeling System (CMAQ) [1] in their current form. For example, during the recent TexAQS 2000 campaign, a wide variety of experimental measurements identified large and frequent Transient High Ozone Events (THOEs), i.e., spikes of ozone, that appear to be directly associated with releases of reactive unsaturated hydrocarbons near the Houston Ship Channel and in adjacent areas [2, 3]. On the other hand, simulations of air quality for the same period of time, using the existing emissions data and the highest resolution of the computational models, often fail to reproduce THOEs. 
CMAQ utilizes a regular grid approach that encounters difficulties in reproducing the ozone productivity measured in the Houston Ship Channel area during the TexAQS 2000 experiment, while a simpler Lagrangian model can be shown to successfully reproducing the observed behavior through the adjustment of input emissions, meteorological conditions, and back-ground chemical conditions. One approach to improve CMAQ is to increase the resolution of both horizontal advection and diffusion through the refinement of the underlying computational mesh, possibly irregular, quadrilateral mesh focused on a sub-domain of a regular rectangular CMAQ grid representing the density of emissions distribution. In the "Static Adaptive Finemesh Eulerian" (SAFE) modeling approach, we utilize the finite difference/volume algorithms on general quadrilateral meshes having the logical, but not necessarily geometric, structure of a regular rectangular grid. The algorithms now being used are extensions of ones that are already used in CMAQ with regular rectangular meshes. The advantages of utilizing SAFE grids are that they limit the computational expense of a highly refined grid to a user defined subdomain of interest and allow a variable degree of refinement inside the chosen subdomain.

\section{Implementation Techniques}

\subsection{CMAQ Enhancements}

The SAFE version of the CMAQ (CMAQ/SAFE) dynamically nests a subdomain with a refined SAFE quadrilateral grid inside of a coarser standard grid called the parent grid. Computations proceed in lock step on both the parent and SAFE grids, with the SAFE grid dynamically receiving interpolated boundary concentrations for tracked species from the latest results on the parent grid. This is dynamic one-way nesting. Normal nesting of CMAQ grids, say a $4 \mathrm{~km}$ grid inside of a $12-\mathrm{km}$ grid, is done with separate executions of CCTM, first on the 12-km grid and then, after a boundary condition file has been generated from the results on the $12 \mathrm{~km}$ grid, the run on the $4 \mathrm{~km}$ grid reads in the boundary condition file. CMAQ/SAFE does dynamic nesting because a future goal is to do two-way nesting, with the run on the refined SAFE grid dynamically feeding its results back to the parent grid. When no initial conditions are available on the SAFE grid, the initial conditions are simply interpolated in space from the initial conditions being used on the parent grid.

To be able to run on a SAFE mesh, "SAFE aware" versions of a number of process subroutines were created. In many cases this just required that the subroutine be modified to process the list of cells in the SAFE grid, rather than in the parent grid. The following briefly describes a list of CMAQ processes modified to create a SAFE version of the associated code:

- Reading External Meteorological and Emission Inputs: Data are read at the parent grid resolution and then interpolated in space to the SAFE grid to provide dynamic boundary conditions.

- Horizontal Mass Transport Processes: Horizontal advection and diffusion algorithms were modified to be "SAFE aware" 
- Vertical Mass Transport Processes: No modification was needed for the vertical advection and diffusion processes because the same horizontal SAFE mesh is used at all vertical layers

- $\quad$ Single Cell Processes: Processes such as Chemistry required modifications to aware the new SAFE data structures.

Other processes, such as Plume-in-Grid modeling, Cloud processes and Aerosol modeling, that are not as significant in modeling ozone production, are not yet supported on SAFE grids.

\subsection{Grid Structures}

The CMAQ/SAFE code and data structures have been designed to work with any horizontal mesh of non-degenerate convex quadrilateral cells that reversibly maps to a regular rectangular grid. This restriction permits use of techniques such as operator splitting that have traditionally been used in air quality modeling on uniform rectangular grids for the irregular grid system. It also simplifies data management tasks and CMAQ's present rectangular data infrastructure based on I/O API can be utilized as is. In principle, if a user supplied the positions of all the nodes of any such a grid, then that data could easily be read into the SAFE data structures and used for a simulation.

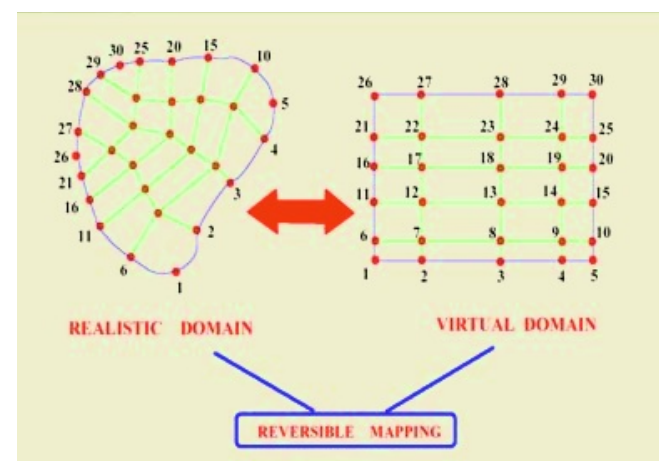

Fig. 1. Demonstration of the structural equivalence of a SAFE grid on an irregular domain with a regular rectangular grid

At this point, a few simple static grid generation algorithms are available as options through CMAQ/SAFE. These algorithms require a very small amount of input data to define the SAFE subdomain and its mesh in terms of the rows and columns of the parent grid. A good deal of work has been done on grid generation algorithms, especially algorithms that automatically adapt the mesh to particular problem and solution features. Investigating such algorithms and incorporating them into CMAQ is a possible area for future work. 


\subsection{Horizontal Transport on a SAFE Mesh}

The governing conservation equation for trace species advection is

$$
\frac{\partial \phi}{\partial t}+\nabla \cdot(\phi \mathbf{V})=0
$$

where $\phi$ is the concentration (as a volume density) of a trace species, and $\mathbf{V}$ is the velocity vector of the wind (again, after transformation to the computing coordinate system). The solution method is the same for both air and the trace species, so we shall present the approach used in terms of a trace species. In order to discretize the advection equation on a SAFE mesh, we consider each quadrilateral SAFE cell to be a control volume. With the approximations that $\phi$ is constant on each cell and that the velocity vector $\mathbf{V}$ is constant along each cell edge, over the duration of a time step $\Delta \mathrm{t}$ we get the equation

$$
\frac{\Delta(\mathrm{A} \phi)}{\Delta \mathrm{t}}=-\left(\mathrm{LE}_{\mathrm{E}} \phi \mathbf{V}_{\mathrm{E}} \cdot \mathbf{V}_{\mathrm{E}-\mathrm{Lw}} \phi \mathbf{V}_{\mathrm{W}} \cdot \mathbf{V}_{\mathrm{W}}\right)-\left(\mathrm{LN}_{\mathrm{N}} \phi \mathbf{V}_{\mathrm{N}} \cdot \mathbf{V}_{\mathrm{N}}-\mathrm{Ls}_{\mathrm{S}} \mathbf{V}_{\mathrm{S}} \cdot \mathbf{V}_{\mathrm{s}}\right)
$$

where $\mathrm{A}$ is the area of the cell, $\mathrm{L}$ is the length of an edge and $\mathbf{V}$ is the unit normal vector to an edge, as shown in Figure 2.

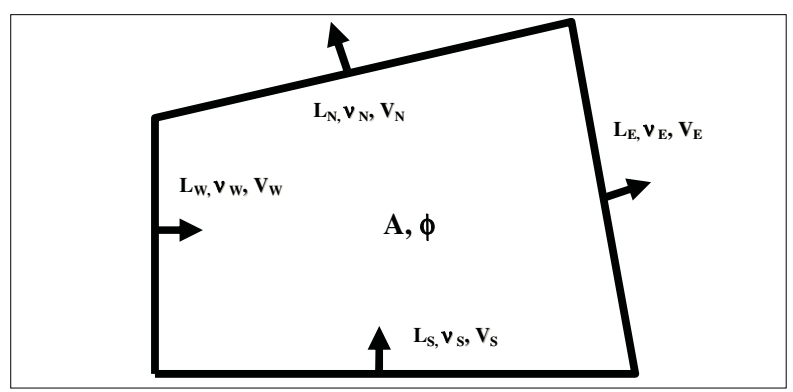

Fig. 2. A typical quadrilateral cell in a SAFE mesh. The arrows show the direction of the unit normal vectors $\mathbf{V}$

By rearranging Equation (2), and splitting it into the (nominal) West-East and SouthNorth directions, we get

$$
\begin{aligned}
& \left.\Delta \phi\right|_{E W}=-\frac{\Delta \mathrm{t}\left(\mathrm{LE} \phi \mathbf{V}_{\mathrm{E}} \cdot \mathbf{V}_{\mathrm{E}}-\mathrm{Lw} \phi \mathbf{v}_{\mathrm{w}} \cdot \mathbf{V}_{\mathrm{w}}\right)}{\mathrm{A}} \\
& \left.\Delta \phi\right|_{N S}=-\frac{\Delta \mathrm{t}\left(\left.\mathrm{LN}_{N} \phi\right|_{E W} \mathbf{v}_{\mathrm{N}} \cdot \mathbf{V}_{\mathrm{N}}-\left.\mathrm{Ls} \phi\right|_{E W} \mathbf{v}_{\mathrm{s}} \cdot \mathbf{V}_{\mathrm{S}}\right)}{\mathrm{A}}
\end{aligned}
$$


With this splitting we can advance the advection solution a time step by sending the first piece for each row of quadrilaterals and the second piece for each column of quadrilaterals to a solver for one dimensional advection. This is done by telling the solver that LV $\bullet \mathbf{V}$ is a speed and A is a "distance" step. The solver has the job of finding a good approximation to the average value of $\phi$ for the area $(\mathrm{LV} \cdot \mathrm{V}) \Delta \mathrm{t}$ that flows through an edge of the cell over a time step. Then $\phi_{a v e} \mathbf{L} \cdot \mathbf{V} \Delta \mathrm{t}$ is the total mass that flows through the edge over the time step. Note that this formulation is guaranteed to conserve mass, but the amount of mass movement between cells is subject to approximation error.

The equation for horizontal diffusion is

$$
\frac{\partial \phi}{\partial t}=\nabla \cdot\left(\rho_{\mathrm{s}} \mathrm{K}_{\mathrm{H}} \nabla \mathrm{q}\right)
$$

where $\rho_{\mathrm{J}}$ is the density of air times the Jacobian determinant of a coordinate transformation, $K_{H}$ is a scalar eddy diffusivity dependent on wind deformation and $q=\phi / \rho$ J is mixing ratio. In $\mathrm{CMAQ} / \mathrm{SAFE}$ this equation is now handled like the advection equation, with the mass flux $\rho_{\mathrm{J}} \mathrm{K}_{\mathrm{H}} \nabla \mathrm{q}$ replacing $\phi \mathbf{V}$. In fact, it is sufficient to use the resulting version of Equation 0 directly, without operator splitting and a higher order approximation method as in the one dimensional advection solver being used.

\section{CMAQ/SAFE Simulation Results}

The domain for our studies of CMAQ/SAFE is shown in Figure 3. This is a domain that contains Houston, the Houston ship channel, Galveston and surrounding areas. For a parent grid with $4 \mathrm{~km}$ cells, the cells in the skewed mesh average out to $2 \mathrm{~km}$ cells, while the smallest cells in the other mesh are $1 \mathrm{~km}$ and the cells in the corner areas are $2 \mathrm{~km}$ cells. Users can choose arbitrary boundary nodes following an irregularly shaped enclosure to adapt the grid to a non-rectangular domain of interest.

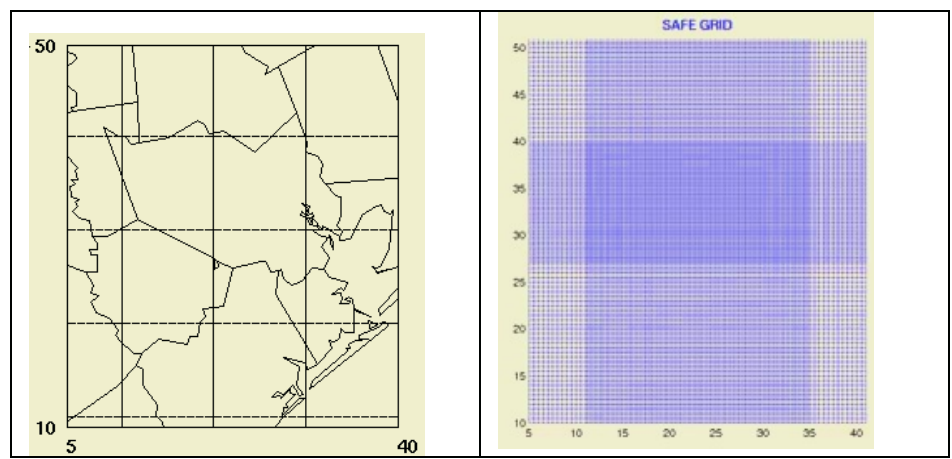

Fig. 3. Subdomain and SAFE mesh used for comparing selected horizontal and vertical results on the parent grid and SAFE grid 


\subsection{Transport Process of CMAQ/SAFE}

Here, we simulated transport processes only, i.e., horizontal and vertical advection and diffusion, using non-reactive tracer species with artificially controlled initial and boundary conditions. The test validates the use of SAFE meshes and the modifications of the advection and diffusion solution algorithms. We compared results (not shown here) for the small subdomains that were obtained with the parent grid's $4 \mathrm{~km}$ mesh, the irregular rectangular SAFE mesh, and the skewed SAFE mesh. The study shows good agreement between the results on the three different grids, with the results on the SAFE grids obviously showing more pronounced local variation. All 3 meshes are coarse enough so that we cannot expect exact agreement. An important thing to notice is that even though the two SAFE grids are distinctly different, the differences in the computed concentrations are quite small. Clearly the choice of mesh, at this degree of refinement, does not change the qualitative results, but does give slightly different local variation in concentrations, which is to be expected.

\subsection{Effects of SAFE Mesh on Nonlinear Chemistry}

We have tested CMAQ/SAFE with transport, emissions and the SAPRC99 gas-phase chemistry mechanism. The parent grid has a 4-km mesh and the SAFE grid used here has a variable rectangular mesh with cell edges either 1-km or 2-km (see Figure 3). It contains 120 columns and 108 rows or 12,960 cells. The grid resolution affects the simulated ozone production because it is produced through the nonlinear chemical reactions of the VOC and $\mathrm{NO}_{x}$ species. When the emissions of either VOC or $\mathrm{NO}_{x}$ species are dispersed in a too larger cell and fail to represent the actual atmospheric conditions, the model would not be successful in generating observed ozone concentrations.

Figure 4 shows $\mathrm{NO}_{\mathrm{x}}$ concentration peaks in the Houston downtown area, with high volume of traffics, thus large $\mathrm{NO}_{\mathrm{x}}$ emissions. SAFE grid provides a more detailed distribution of $\mathrm{NO}_{\mathrm{x}}$. However, because the road network is wide spread over the large downtown area, there is not much difference in the peak values. On the other hand, when highly reactive VOC species are emitted in a small but concentrated industrial area, such as the Houston Ship Channel, the model grid should be sufficiently small to properly describe $\mathrm{NO}_{\mathrm{x}}$-VOC concentration ratios to replicate real atmospheric conditions. Figure 4 also contrasts the resulting ozone concentration differences. The CMAQ/SAFE with higher grid resolution can successfully simulate the high ozone event in the Houston. It is evident in Figure 5 that the high ozone concentration from the CMAQ/SAFE resulted from the higher HCHO concentrations, which in turn was produced through the photo-oxidation of the highly reactive VOC emissions from the Ship Channel industries. Aircraft measurements during the TexAQS studied showed the presence of high $\mathrm{HCHO}$ and ozone plumes in around and downwind of the Houston Ship Channel area. Only when the $\mathrm{NO}_{\mathrm{x}}$ VOC concentration ratios are properly represented, the model can simulate the high ozone concentrations observed. 


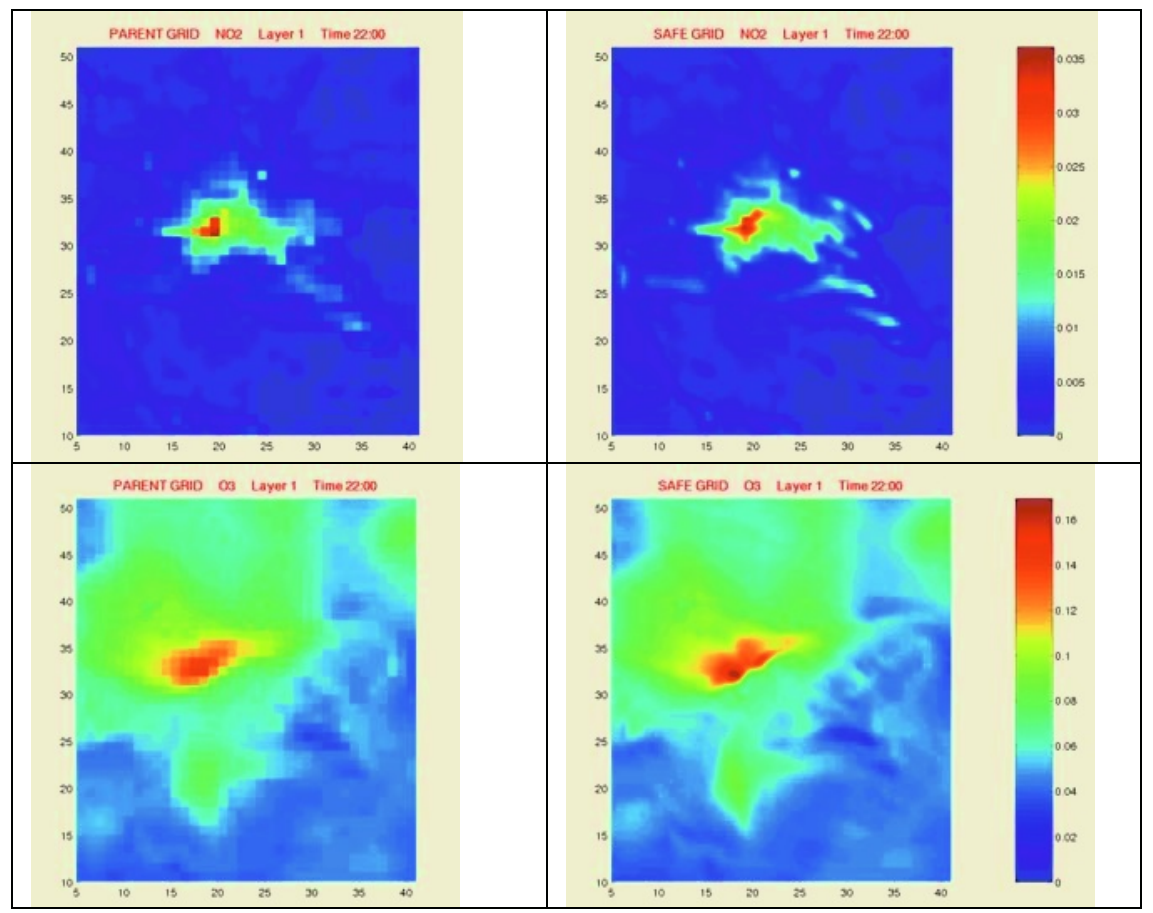

Fig. 4. Comparison of $\mathrm{NO}_{2}$ (top) and $\mathrm{O}_{3}$ (bottom) concentrations between the parent grid (left) and SAFE grid (right)

\section{Conclusions}

The goal of this study was to implement CMAQ the capability to efficiently focus computing resources for enhanced resolution of its science processes in geographical areas where the user needs an extra degree of detail. The SAFE grid in the target sub-window is a more refined and more flexible grid, e.g., with rectangular, or even quadrilateral cells, whose shape and size can vary within the grid. The solution on the SAFE grid provides more detail inside its sub-window, but currently does not improve the solution on the parent grid. At this time, many of the science processes have been extended to work simultaneously on a regular "parent" grid and a SAFE grid on a single targeted sub-window. Further enhancement tasks under development are; (1) two-way nesting solution to utilize the refined sub-grid results feed back to the parent grid, and (2) multiple SAFE domains inside the same parent grid. For example, this capability could be used to improve modeling of the air quality interaction between two large, but separate, cities, such as Houston and Dallas within Texas. The two-way nesting capability described above is needed to realize an improvement in the interaction between improved solutions in multiple SAFE windows. 


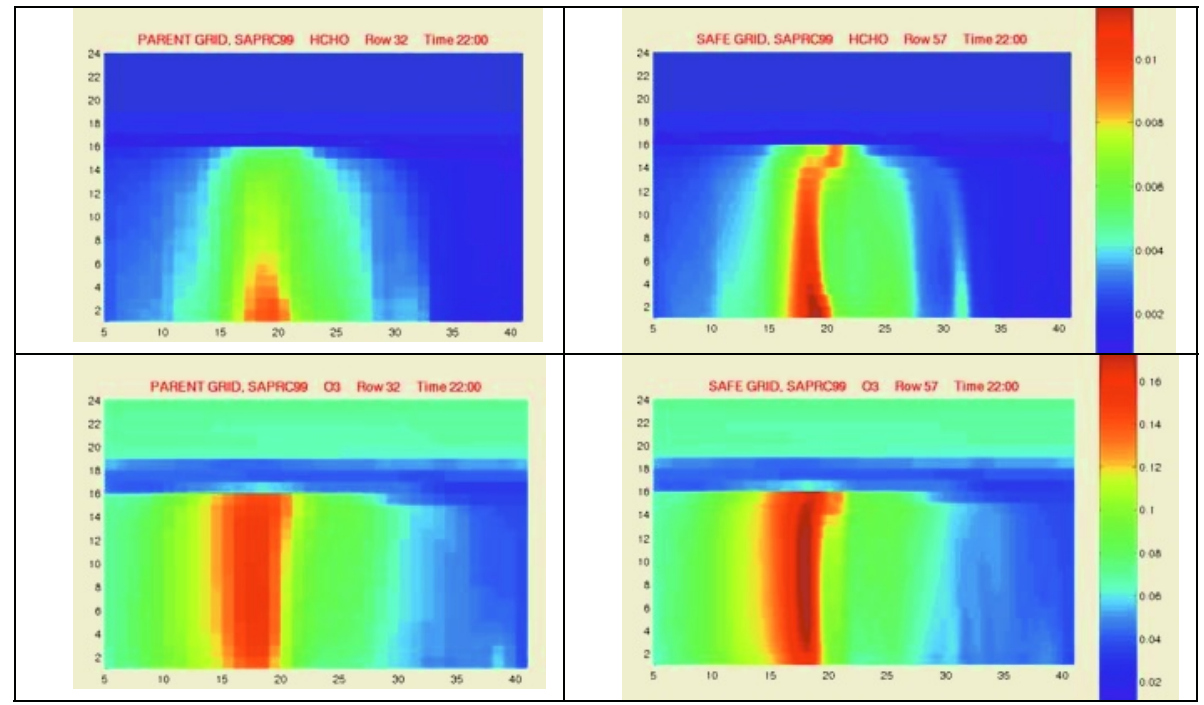

Fig. 5. Vertical cross section over the Ship Channel area of $\mathrm{HCHO}$ (top) and $\mathrm{O}_{3}$ (bottom) for the parent mesh (left) and the SAFE mesh (right)

\section{References}

1. Byun, D.W. and Ching, J.K.S.: Science algorithms of the EPA Models-3 Community Multiscale Air Quality (CMAQ) Modeling System". EPA-600/R-99/030, U.S. EPA. (1999) (available at http://www.epa.gov/asmdnerl/models3/doc/science/science.html )

2. Byun, D.W., Kim, S.-T., Cheng, F.-Y., Kim, S.-B., Cuclis, A., and Moon, N.-K.: Information Infrastructure for Air Quality Modeling and Analysis: Application to the HoustonGalveston Ozone Non-attainment Area, J. Environmental Informatics, 2(2) (2003) 38-57

3. Daum, P.H., L. I. Kleinman, S. R. Springston, L. J. Nunnermacker, Y.-N. Lee, J. Weinstein-Lloyd, J. Zheng, and C. M. Berkowitz: A comparative study of O3 formation in the Houston urban and industrial plumes during the 2000 Texas Air Quality Study. Journal of Geophysical Research, 108(D23) (2003) 4715, doi:10.1029/2003JD003552. 